

\title{
ESTRATÉGIA PARA A INCORPORAÇÃO DE IMPACTES AMBIENTAIS, SOCIAIS E ECONÓMICOS ESPECÍFICOS NUM MÉTODO DE AVALIAÇÃO DA SUSTENTABILIDADE DE EDIFÍCIOS DE SAÚDE (HBSA METHOD)

A STRATEGY FOR THE INCORPORATION OF SPECIFIC ENVIRONMENTAL, SOCIAL AND ECONOMIC IMPACTS INTO A HEALTHCARE BUILDING SUSTAINABILITY ASSESSMENT (HBSA) METHOD

\author{
MARIA DE FÁTIMA CASTRO \\ Universidade do Minho \\ Guimarães, Portugal \\ info@mfcastro.com \\ RICARDO MATEUS \\ Universidade do Minho \\ Guimarães, Portugal \\ ricardomateus@civil.uminho.pt
}

\author{
LUÍS BRAGANÇA \\ Universidade do Minho \\ Guimarães, Portugal \\ braganca@civil.uminho.pt
}

RESUMO

O projeto de um ambiente hospitalar requer uma série de preocupações com a satisfação e bemestar da equipa de trabalho, pacientes e administradores, ao mesmo tempo que possui uma forte responsabilidade social e impacte na cidade. Atualmente, verifica-se que a maioria deste tipo de edifícios não é concebida ou utilizada de forma sustentável, apesar de já existirem algumas ferramentas que têm vindo a ser desenvolvidas com o objetivo de serem utilizadas no suporte de decisões que permitam otimizar a sustentabilidade dos mesmos. Tendo por base este contexto, o presente artigo tem como objetivos discutir as barreiras existentes na disseminação de práticas sustentáveis nos edifícios de saúde e apresentar uma proposta de estrutura para uma metodologia que, dando resposta a algumas das barreiras identificadas, facilite a incorporação de princípios de sustentabilidade durante as diferentes fases do ciclo de vida de um edifício hospitalar. A estrutura apresentada é orientada para o contexto português e encontra-se baseada no trabalho desenvolvido até então pelas organizações ISO e CEN e noutras metodologias internacionais de avaliação da sustentabilidade já disponíveis no mercado.

Palabras clave

métodos de avaliação, método HBSA, edifícios de saúde, hospitais, sustentabilidade

\section{ABSTRACT}

The design of a hospital environment involves many concerns related to the satisfaction and wellbeing of the medical personnel, patients, and administrators, and at the same time requires strong social responsibility and has a high impact on a city. Currently, it seems that the majority of this type of building is not designed or used in a sustainable way, although some tools have already been developed to support decision-making to optimize building sustainability. Based on this context, the aim of this article is to discuss the barriers to the dissemination of sustainable practices in healthcare

buildings, and to propose the structure of a methodology that facilitates the incorporation of sustainability principles during the different life-cycle stages of a hospital building while overcoming some of the identified barriers. The structure presented is oriented to the Portuguese context and is based on the work done to date by the ISO and CEN organizations and other international sustainability assessment schemes already available on the market. 


\section{INTRODUÇÃO.}

\section{ENQUADRAMENTO.}

As problemáticas e condicionantes associadas aos edifícios de saúde, variam de continente para continente e até mesmo de país para país. Por exemplo, no contexto Europeu sabese que, aproximadamente, $10 \%$ do PIB de cada país é utilizado em edifícios orientados para os cuidados médicos e, de acordo com os dados de 2008, estes edifícios acabam por contribuir com, pelo menos, $5 \%$ das emissões totais de $\mathrm{CO}_{2}$ dos países da União Europeia (ASHE, 2002; Vaquero, 2013). Por sua vez, de acordo com U.S: Energy Information Administration, durante o ano de 2007 nos E.U.A, o setor foi responsável por $5,5 \%$ do total da energia consumida pelo setor comercial, tendo-se constatado um aumento em relação aos 4,3\%, verificados em 2003 (ASHE, 2002; Pentland, 2012). Em comparação com outros sectores, o sector da saúde apresenta uma evolução muito dinâmica, pautada pelo aparecimento de novos equipamentos e técnicas de diagnóstico e tratamento de doenças, pelo que o seu impacte nas três dimensões do desenvolvimento sustentável tem vindo a aumentar. Desta forma, é cada vez mais relevante a existência e aplicação de sistemas de avaliação da sustentabilidade no contexto do sector da saúde.

O projeto, construção e utilização sustentáveis de um edifício, baseiam-se no equilíbrio entre: as questões ambientais (relacionadas com o impacte ambiental); as características sociais (relacionadas com o conforto dos utilizadores e com outras questões sociais); e os aspetos económicos (associados ao custo do ciclo de vida). Assim, o projeto sustentável de um edifício, analisado como um todo, procura a melhor compatibilidade possível entre o ambiente construído e o natural, não comprometendo os requisitos funcionais e respetivos custos associados para os quais os edifícios são projetados.

Este objetivo é a base das principais metodologias de avaliação da sustentabilidade existentes, assim como dos documentos orientadores que têm vindo a ser desenvolvidos com a finalidade de apoiar tanto as equipas de projeto como as de gestão dos edifícios. Neste contexto, têm surgido várias questões: será que estas metodologias consideram as especificidades dos edifícios de saúde? Incorporarão elas, verdadeiramente, os impactes específicos deste tipo de edifícios nas três dimensões do Desenvolvimento Sustentável? Quais são as principais preocupações consideradas na conceção dos reconhecidos casos de estudo? As respostas a estas questões serão apresentadas e discutidas ao longo deste artigo.

As mais reconhecidas iniciativas de desempenho ambiental que cedo se fizeram notar, focaram-se especialmente na preocupação com a redução do consumo de energia. Assim, diferentes instituições e iniciativas governamentais desenvolveram ferramentas e diretrizes orientadas para este problema.
Nos anos 80 e 90 do século XX, algumas destas iniciativas começaram a incorporar preocupações sustentáveis, no que diz respeito à industria de construção, e em 1993 o UIA/AIA Word Congress for Architects concluiu que era um desafio prioritário para os profissionais de arquitetura porem em prática os princípios da sustentabilidade nos seus processos de projeto (Guenther e Vittori, 2008).

No ano 2000 muitas das iniciativas passaram a incorporar práticas sustentáveis de projeto como a base e fundamento do pensamento do processo arquitetónico e projetual. Em 2005 o American Institute of Architects (AIA), estabeleceu a posição mais firme relativamente às responsabilidades dos profissionais de arquitetura, defendendo que estes deveriam alterar as ações dos processos de projeto e trabalharem juntamente com os clientes para que se conseguisse alterar o atual paradigma no que respeita à falta de ligação entre o processo de projeto e o de utilização de um edifício (Hamilton, 2006).

O projeto sustentável requer uma verdadeira revolução na maneira de pensar o projeto de um edifício, por isso torna-se fundamental que esta transformação, a qual atravessa todas as fases do ciclo de vida de um edifício, seja refletida na fase inicial do projeto de arquitetura, essencialmente na fase de organização e conceção espacial.

Se o projeto de arquitetura deve conter as necessidades especificas dos pacientes e utilizadores de um edifício hospitalar, as preocupações ambientais e ainda gerar sinergias entre todos os intervenientes no processo de construção de um edifício desta tipologia, então estes princípios deverão ser considerados nas ferramentas de avaliação da sustentabilidade dos edifícios de saúde. Isto torna-se essencial para apoiar os profissionais que desenvolvem estes projetos, a fim de os apoiar desde a fase inicial de pensamento do projeto de arquitetura e também para reconhecer os esforços que foram desenvolvidos no sentido de construção de um edifício mais sustentável.

Michael Lerner (2000) lançou a seguinte questão: "A questão é se os profissionais de saúde estão a começar a reconhecer as consequências ambientais das nossas ações e têm vontade de colocar esta casa em ordem?" (Roberts e Guenther, 2006: 82). Esta não é uma pergunta trivial, mas sim a base de todas as outras questões que possam ser levantadas em torno desta mesma preocupação. Com base neste princípio, a Figura 1 ilustra a relação entre a saúde humana, o tratamento médico e a poluição ambiental, aspetos que afetam diretamente a missão da indústria de cuidados de saúde. 


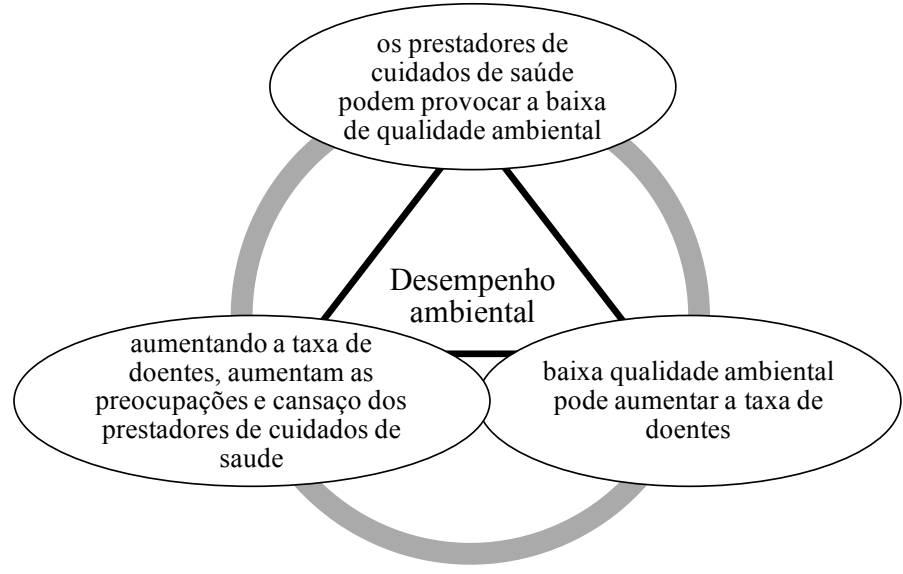

Figura 1. Relação existente entre poluição ambiental e cuidados de saúde (Roberts e Guenther, 2006:83).

\section{BARREIRAS E BENEFÍCIOS ASSOCIADOS ÀS PRÁTICAS DE SUSTENTABILIDADE NOS EDIFÍCIOS DE SAÚDE.}

O processo de definição dos benefícios, tanto para a organização, como para a comunidade, resultantes da introdução das práticas sustentáveis de projeto no setor da saúde, continua a ser um desafio. Assim, torna-se importante que estes benefícios sejam definidos e quantificados, assim como comunicados e rapidamente compreendidos por todos os intervenientes do sector.

Os benefícios resultantes da aplicação de práticas sustentáveis que visam a "eficiência" e "redução" são geralmente mal interpretados pelos utilizadores dos edifícios de saúde, uma vez que este sector trata das pessoas e da sua qualidade-devida e bem-estar (Guenther e Vittori, 2013). Paralelamente, as organizações de saúde têm vindo a reunir esforços, no sentido de se alcançarem e explicarem os benefícios sociais da implementação de estratégias de redução do impacte ambiental destes edifícios. Inúmeras organizações e edifícios de saúde têm vindo a ser reconhecidos pelas suas iniciativas: tratamento de resíduos hospitalares; programas de reciclagem; programas de melhoria do impacte ambiental que têm reflexo nas comunidades vizinhas; redução do consumo de energia; redução do consumo de água; etc.

Assim, neste contexto, é fundamental promover o diálogo entre os princípios fundamentais de projeto desta tipologia de edifícios e as estratégias de introdução de práticas sustentáveis na indústria de saúde. A maior distinção que se pode fazer sobre estes dois conceitos é que: os princípios fundamentais de projeto são fruto da investigação focada nas preocupações médicas e no objetivo definido para cada espaço de trabalho que incorpora as recomendações de ambiente controlado que este tipo de edifícios deve manter; por seu lado, as práticas sustentáveis de projeto são um processo em desenvolvimento que definem um determinado número de critérios para melhoria do impacte ambiental do edifício, com base em considerações, normas e estudos que estão em constante desenvolvimento (Hamilton 2006). As estratégias tomadas pela implementação das práticas sustentáveis de projeto têm em consideração a saúde pública, a comunidade e as preocupações sociais.

Robert e Guenther definem como "Triple Bottom Line for Health" o entendimento feito pelo sector da saúde acerca da sustentabilidade dos edifícios e da sua exequibilidade e que assenta nos três seguintes pilares (Roberts e Guenther, 2006): desempenho económico; desempenho ambiental; e desempenho social/ético do sucesso de cada unidade de atendimento de saúde. Esta é a base das mais reconhecidas ferramentas de avaliação da sustentabilidade que têm vindo a ser desenvolvidas com o propósito de reconhecer as organizações e os projetistas que têm em conta estas preocupações nas suas intervenções. Contudo, estas considerações aumentam de complexidade quando se acrescentam os interesses da comunidade e da população que podem induzir à ineficácia ou aplicação incorreta das mesmas. Neste sentido, a indústria de saúde deve fazer um esforço para incorporar não só as exigências técnicas dos hospitais, dos pacientes e do ambiente, como também da comunidade como um todo.

Shepley and Baum (2009) têm estudado os conflitos existentes entre os dois seguintes conceitos: Eco-Effective Design (EED); Evidence-Based Design (EBD). Embora os seus princípios possam estar assentes nas mesmas bases e numa mesma estrutura, existem conflitos entre estes dois conceitos que se prendem, por exemplo, com a conceção e construção de quartos maiores para os pacientes (Baum et al., 2009b). Por um lado, esta opção pode aumentar o conforto do paciente, possibilitando-lhe mais espaço e melhores condições. Por outro lado, este aumento de área levará a uma maior utilização de recursos construtivos, aumentará o consumo de água e energia para possibilitar uma boa iluminação e refrigeração ou aquecimento do ar. Assim, mais de $50 \%$ de especialistas em EED e EBD defendem que estes dois conceitos devem caminhar juntos e com um mesmo objetivo, promovendo-se e complementando-se (Baum e Shepley, 2009). 


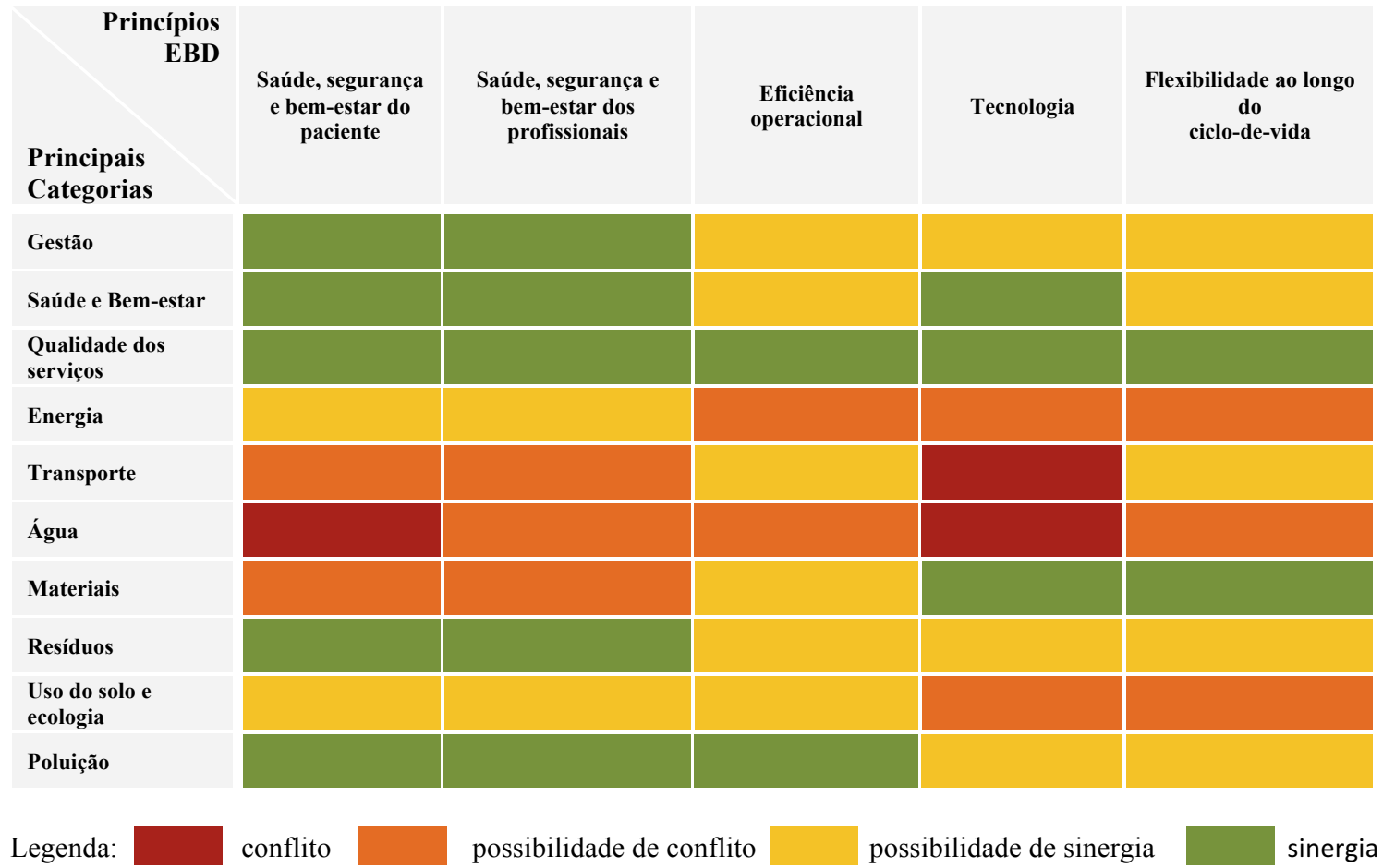

Figura 2. Relação existente entre os princípios defendidos pelo conceito EBD e as principais categorias das metodologias de avaliação da sustentabilidade dos edifícios hospitalares (Castro, Mateus e Bragança, 2014:610).

\section{EVOLUÇÃO E INTEGRAÇÃO DOS ATUAIS PARADIGMAS DE PROJETO: SUSTAINABLE-EFFECTIVE DESIGN (SED) VERSUS EVIDENCE-BASED DESIGN (EBD).}

A relação entre os dois conceitos já anteriormente apresentados, Evidence-Based Design (EBD) e Eco-Effective Design (EED), tem vindo a ser discutida, estudada e apresentada, conforme literatura revista (Baum et al., 2009a). No entanto, pode dizerse que no conceito de EED não cabem todas as preocupações inerentes ao conceito de construção sustentável. Assim, torna-se fundamental a introdução de um novo conceito, Sustainable-Effective Design (SED), capaz de refletir todas as questões que devem ser tidas em conta nas metodologias de avaliação da sustentabilidade dos edifícios hospitalares. A maioria dos estudos que se focam no conceito EED procuram uma resposta para a conceção de um edifício com o mínimo impacte ambiental possível, para que seja gerado um "healing environment". Tendo em conta este contexto, a Figura 2 reflete as sinergias e conflitos entre as estratégias apontadas e defendidas pelo conceito EBD e as principais categorias das metodologias de avaliação da sustentabilidade em estudo neste artigo.

Salvo raras exceções, como as categorias "Água" e "Transporte", todas as restantes categorias estão intrinsecamente relacionadas com as estratégias defendidas pelo conceito EBD. A maior parte das categorias apresentam possibilidade de sinergia ou total sinergia com os princípios defendidos pelo conceito EBD, sendo de realçar a categoria "Qualidade dos Serviços" como a que apresenta total sinergia com todos os princípios defendidos.

Para um melhor entendimento desta relação, todos os indicadores de cada categoria deveriam ser tidos em conta numa análise comparativa como a da Tabela 1, a fim de se compreender quais os indicadores que seguem a par ou não com os princípios defendidos pelo EBD. Da mesma forma, as melhores práticas do EBD deveriam ser tidas em conta nesta análise. Assim, é importante salientar que se torna importante ter em conta estas sinergias e conflitos para melhorar a compreensão entre estas duas realidades, a fim de se começar a pensar no conceito SED como aglutinador de todos estes princípios.

Um dos objetivos destas metodologias é ajudar as equipas projetistas na fase de projeto e construção. Assim, a combinação entre os princípios defendidos pelo conceito EBD e as estratégias do conceito EED, é importante para se conseguir alcançar os indicadores que poderão enquadrar o conceito SED a fim de este ser claramente considerado nas metodologias de avaliação da sustentabilidade em constante desenvolvimento. 


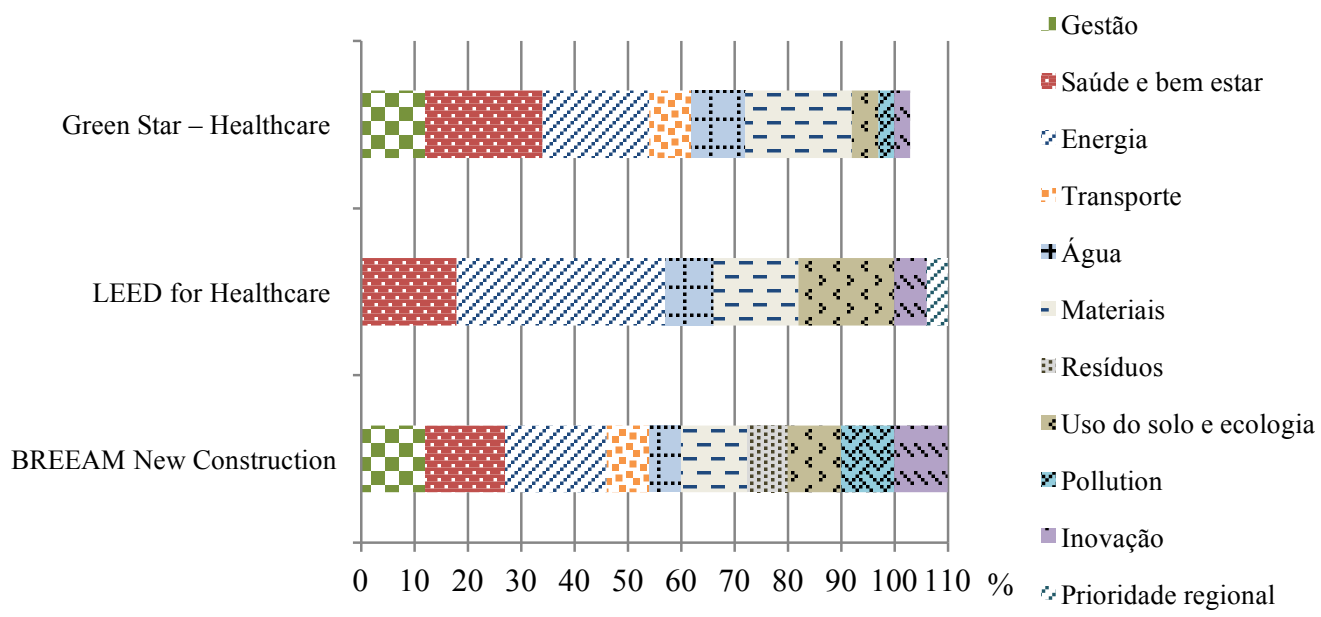

Figura 3. Distribuição de pesos nas metodologias de avaliação BREEAM New Construction, LEED for Healthcare e Green Star - Healthcare (elaborada pelo autor).

\section{AVALIAÇÃO DA SUSTENTABILIDADE NO CONTEXTO DOS EDIFÍCIOS DE SAÚDE.}

Com o intuito de promover a introdução de práticas sustentáveis no projeto e utilização de edifícios hospitalares, alguns países, organizações e associações têm vindo a publicar algumas diretrizes neste domínio. De entre elas são de destacar as recomendações para projetos hospitalares que o Green Building Committee da American Society of Healthcare Engineering (ASHE) publicou em 2002. Pensando em melhoraro meio ambiente, a American Hospital Association's, juntamente com a United States Environmental Protection Agency, propôs, através dos princípios da arquitetura sustentável, regras para reduzir os resíduos e outros impactes associados aos hospitais (ASHE, 2002).

Nessas recomendações, a ASHE propõe um desenvolvimento arquitetónico e construtivo capaz de melhorar as preocupações a nível da saúde em três escalas (ASHE 2002):

proteção da saúde de todos os utilizadores dos edifícios; proteção da saúde da comunidade vizinha;

proteção da saúde da comunidade global e dos recursos naturais.

Neste sentido, há um número significativo de metodologias de avaliação da sustentabilidade, orientadas para o sector da construção, que têm como foco a construção nova, os edifícios existentes e/ou a reabilitação e manutenção dos mesmos. Dentro destas metodologias, alguns sistemas têm vindo a orientar-se também para o sector da saúde, procurando desenvolver ferramentas específicas para a avaliação desta tipologia de edifícios. Analisando o estado da arte destas metodologias, é possível salientar as seguintes, pela sua maior presença e afirmação a nível mundial: BREEAM New Construction; LEED for Healthcare; Green Star - Healthcare; and CASBEE for New Construction (Castro, Mateus e Bragança, 2015a)

\section{CARACTERIZAÇÃO DAS METODOLOGIAS DE AVALIAÇÃO EXISTENTES.}

As metodologias de avaliação da sustentabilidade de edifícios de saúde, apresentam no geral uma estrutura semelhante. São todas compostas por categorias (as quais identificam as principais áreas de avaliação), e indicadores (que possibilitam a avaliação do comportamento das soluções escolhidas em relação a diferentes objetivos do conceito de desenvolvimento sustentável) e permitem fazer a avaliação e ponderação de cada ponto que se considera relevante para a avaliação da sustentabilidade da construção dos edifícios hospitalares.

Segundo Lee (Lee et al., 2002), a estrutura é a base de todos os sistemas de avaliação, uma vez que é esta que estabelece e permite ponderar a classificação final do desempenho global do edifício. No entanto, ainda não existe unanimidade no que diz respeito à ponderação feita nestes sistemas de avaliação. Assim, cada sistema de avaliação da sustentabilidade considera no cálculo do desempenho global um diferente sistema de pesos para a ponderação do desempenho obtido ao nível de cada indicador de sustentabilidade.

Na Figura 3 apresentam-se os diferentes pesos atribuídos a cada uma das categorias de sustentabilidade pelas metodologias em análise. No que respeita às metodologias BREEAM New Construction, LEED for Healthcare e Green Star - Healthcare, estas apresentam para além de uma estrutura semelhante, um sistema de ponderação de pesos idêntico, embora o peso atribuído a cada categoria seja diferente. Quanto à metodologia CASBEE for New Construction - Hospitals, não é possível compará-la com as outras metodologias uma vez que a sua estrutura assenta em dois principais grupos de indicadores [qualidade ambiental do edifício (Q) e impacte ambiental do edifícios $(L R)]$ e não têm correspondência com a das outras metodologias. Adicionalmente, o peso final destas duas categorias varia de acordo com as pontuações atribuídas aos indicadores intermédios. 


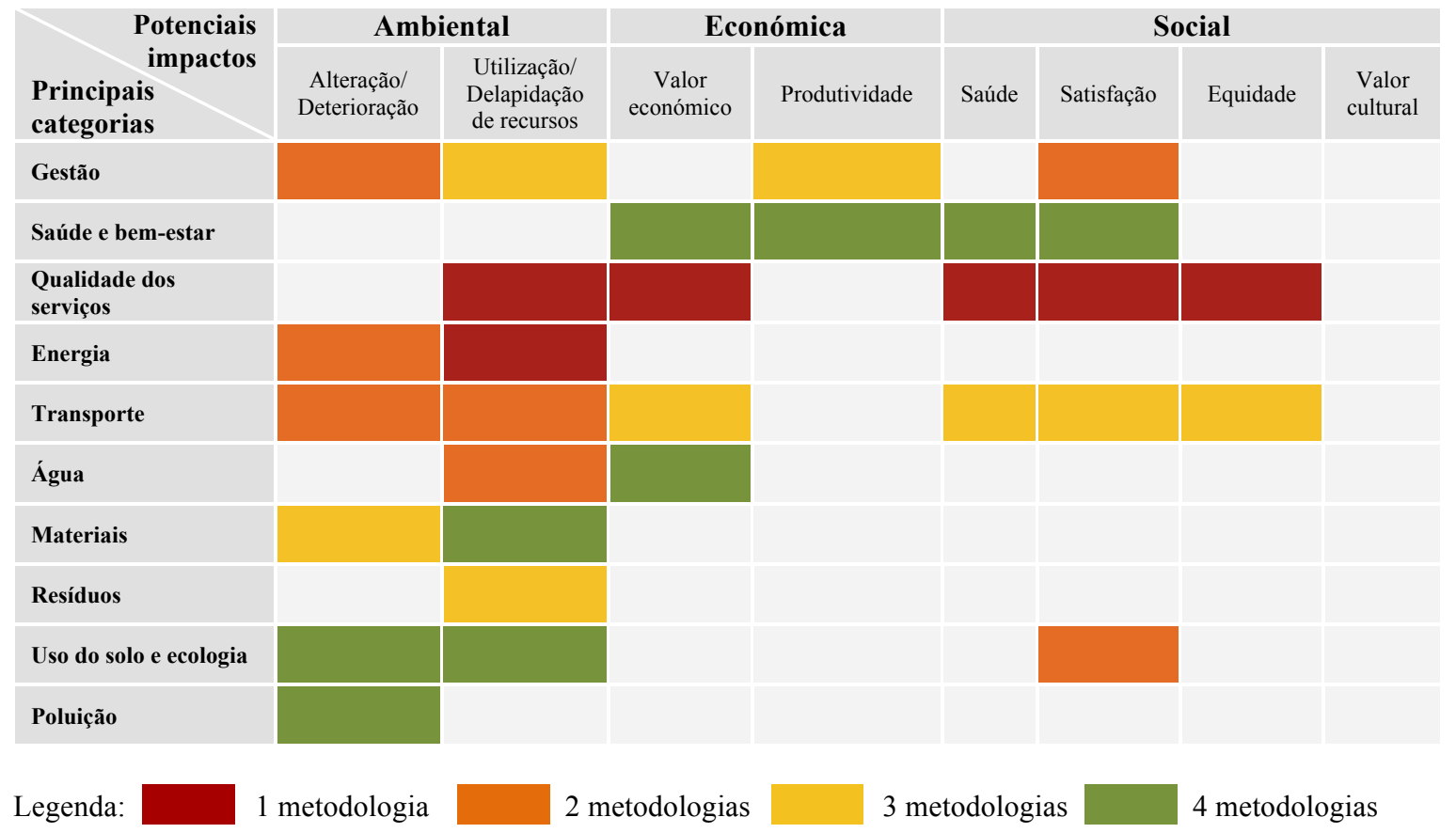

Figura 4. Relação entre os potenciais impactes determinados pela norma ISO/AWI 21929 e as principais categorias definidas pelas metodologias de avaliação da sustentabilidade dos edifícios de saúde estudadas (Castro, Mateus e Bragança 2015b:610)

Analisando a Figura 3, verifica-se que as metodologias BREEAM New Construction e Green Star - Healthcare aproximam-se quanto às categorias de sustentabilidade que contemplam. No entanto, em termos de ponderação (sistema de pesos), esta última tende a assemelhar-se ao LEED for Healthcare, onde as categorias "Energia" e "Saúde e bem-estar" contêm mais de $50 \%$ dos créditos, enquanto que as mesmas categorias no BREEAM New Construction apresentam um peso de apenas $30 \%$. Por sua vez, a metodologia BREEAM New Construction destaca-se por possuir uma distribuição mais equilibrada dos pesos e por conter um maior número de categorias principais de sustentabilidade principais, seguida do Green Star Healthcare.

Nas metodologias LEED for Healthcare e Green Star Healthcare é ainda de ressaltar a existência de duas categorias (Inovação e Prioridade Regional), que premeiam o nível de inovação incorporado no projeto e a prioridade regional dada a cada uma das restantes categorias. Isto é, estas duas categorias permitem um reconhecimento adicional para um edifício que inove no campo do desenvolvimento sustentável e se preocupe com a promoção e sustentabilidade da região apresentando um desempenho acima e além do nível que é atualmente reconhecido e recompensado pela metodologia em questão. A satisfação dos indicadores desta categoria permite um aumento de $10 \%$ no resultado da avaliação global do edifício (Figura 3).

\section{NORMAS EXISTENTES E EM DESENVOLVIMENTO.}

Nos últimos anos, a International Organization for Standardization (ISO) e o European Committee for Standardization (CEN) têm vindo a tentar estabelecer uma definição para o conceito de "construção sustentável". Como resultado existem várias normas já publicadas por ambos: ISO 15392; ISO/IEC TS 17021-4; ISO 20121; ISO 21929-1; ISO 21930; ISO 21931-1; ISO/TR 21932; EN 15643; EN 15942; EN 15804; EN 15978; EN 16309; e EM 16627.

Analisando estas normas é possível concluir que construção sustentável não significa apenas melhorar e defender o meio ambiente, mas também promover e estabilizar o equilíbrio entre este e os aspetos sociais e económicos. Neste contexto, a Figura 4 apresenta a relação entre as categorias definidas pelas metodologias acima referidas e estudadas e as três dimensões da sustentabilidade (e os seus potenciais impactes), de acordo com a divisão proposta pela norma ISO/AWI 21929 (ISO TS 2010).

Tendo por base os resultados apresentados na Figura 4, é possível concluir que a dimensão ambiental é a que possui maior peso e presença em todas as categorias. Por sua vez, é ainda possível ressaltar que estas quatro metodologias em análise, consideram de forma diferente as três dimensões do desenvolvimento sustentável. 


\section{PROPOSTA PARAAESTRUTURADEUMA NOVA METODOLOGIA DE AVALIAÇÃO DA SUSTENTABILIDADE DE EDIFÍCIOS DE SAÚDE PORTUGUESES - HBSATOOL - PT.}

\section{CONTEXTO PORTUGUÊS.}

Em Portugal, o Ministério da Saúde realizou, em 2008, um documento que enumera as recomendações e especificações técnicas dos edifícios hospitalares. Neste documento reeditado em 2012 (ACSS, 2012), encontram-se detalhadas diversas especialidades, como a arquitetura, instalações e equipamentos de águas e esgotos, sistemas elétricos e mecânicos, gestão técnica centralizada, espaços exteriores, gestão integrada de resíduos, manutenção, etc. A par deste tipo de documentos, existem outros regulamentos que especificam as exigências de cada espaço específico ao nível de iluminação, qualidade do ar interior, temperatura, ventilação, etc. No entanto, no que respeita à gestão sustentável dos edifícios hospitalares não existe nenhum documento com força de lei, ou de recomendação.

Em 2013, o Ministério da saúde publicou o "Guia de boas práticas para o setor da saúde", o qual apresenta-se como um instrumento de promoção da redução do consumo de energia, de água, de produção e gestão de resíduos e a disseminação e promoção de comportamentos que promovam economias de baixo carbono. Este guia pode ser utilizado como uma ferramenta de disseminação e divulgação das melhores práticas a todos os utilizadores dos edifícios de saúde. A ideia foi a de demonstrar medidas que exigem custos muito baixos de investimento e a importância de se começar a introduzir tais preocupações nesta tipologia de edifícios (ACSS, 2013).

\section{APRESENTAÇÃO DA ESTRUTURA.}

Não existe uma simples resposta que se possa dar à seguinte pergunta: "O que faz um edifício de saúde ser sustentável?". Isto acontece porque a sustentabilidade não é um conceito que se possa medir e qualificar facilmente (Mateus 2009). No entanto, as metodologias de avaliação da sustentabilidade tentam ajudar a encontrar uma resposta cada vez mais clara para esta pergunta. Assim, elas necessitam de ser o mais abrangentes possível, mas também ser simultaneamente fáceis de entender e de serem aplicadas.
A estrutura da metodologia de avaliação da sustentabilidade que se propõe, permite avaliar e classificar o desempenho de um edifício de saúde relativamente a dois níveis de referência, prática convencional e melhor prática (adaptados ao contexto português), e tem como principais objetivos:

Ser adaptada à realidade portuguesa tendo por base as metodologias mais conhecidas a nível mundial no contexto dos edifícios de saúde, os casos de estudo mais proeminentes e o trabalho desenvolvido pelas organizações ISO e CEN;

Incluir as três principais dimensões do Desenvolvimento Sustentável e permitir a identificação rápida das áreas a avaliar; Apresentar uma lista de indicadores suficientemente extensa (incluindo os impactes mais importantes) ao mesmo tempo que o mais reduzida possível a fim de potenciar a sua utilização;

Permitir a coexistência entre critérios quantitativos e qualitativos, tentando reduzir ao máximo a subjetividade destes últimos, aumentando a fiabilidade dos resultados;

Desenvolver um sistema de avaliação e um certificado facilmente entendível por todos os intervenientes no setor dos edifícios hospitalares.

Esta estrutura de avaliação assenta em critérios de avaliação, repartidos por cinco Áreas, vinte e duas Categorias e cinquenta e dois Indicadores, referentes à fase de conceção de um edifício de saúde novo, ou às fases de utilização e/ou reabilitação de um edifício existente (Tabela 1). A classificação final deste sistema é apresentada através de uma escala de qualitativa (de $A+$ a E), sendo necessária a satisfação de todos os requisitos obrigatórios de acordo com a legislação em vigor em Portugal. Neste momento, a metodologia de avaliação ainda se encontra em fase de desenvolvimento e contemplará um guia de avaliação que apoiará as avaliações de sustentabilidade ao nível de cada uma das cinco Áreas contempladas (Figura 5): A1) Ambiental; A2) Sociocultura e Funcional; A3) Económica; A4) Técnica; A5) Lugar. Tal como apresentado na Figura 5, a metodologia de avaliação assenta em quatro etapas: quantificação; normalização; agregação; e avaliação do desempenho e comunicação. Cada uma destas etapas é apresentada de forma resumida nas secções seguintes. 


\begin{tabular}{|c|c|c|}
\hline ÁREA & CATEGORIA & INDICADOR \\
\hline \multirow{16}{*}{ A1: AMBIENTAL } & C1: Avaliação do impacte ambiental de ciclo de vida & 11: Impactes ambientais do ciclo de vida \\
\hline & \multirow{2}{*}{$\begin{array}{l}\text { C2: Avaliação do impacte ambiental de ciclo de vida - } \\
\text { Energia primária }\end{array}$} & 12: Energia primária não renovável \\
\hline & & 13: Produção local de energia \\
\hline & \multirow[b]{7}{*}{ C3: Uso do solo e biodiversidade } & 14: Otimização da implantação \\
\hline & & I5: Impermeabilização do solo \\
\hline & & 16: Reutilização de áreas previamente utilizadas \\
\hline & & 17: Proteção ecológica do lugar \\
\hline & & 18: Reabilitação da envolvente \\
\hline & & 19: Uso de plantas autóctones \\
\hline & & I10: Efeito de ilha de calor \\
\hline & \multirow[b]{3}{*}{ C4: Materiais e resíduos sólidos } & $\begin{array}{l}\text { 111: Aquisição responsável de produtos de base } \\
\text { orgânica }\end{array}$ \\
\hline & & I12: Separação de resíduos \\
\hline & & 113: Tratamento de resíduos tóxicos \\
\hline & \multirow[b]{3}{*}{ C5: Água } & 114: Consumo de água potável \\
\hline & & I15: Reciclagem e aproveitamento de efluentes \\
\hline & & I16: Tratamento de efluentes contaminados \\
\hline \multirow{16}{*}{$\begin{array}{c}\text { A2: } \\
\text { SOCIOCULTURAL } \\
\text { E FUNCIONAL }\end{array}$} & \multirow[b]{6}{*}{ C6: Conforto e saúde dos utilizadores } & $\begin{array}{l}\text { 117: Eficiência da ventilação natural em espaços } \\
\text { interiores }\end{array}$ \\
\hline & & 118: Toxicidade dos materiais de acabamento \\
\hline & & I19: Conforto Térmico \\
\hline & & 120: Conforto Visual \\
\hline & & 121: Conforto Acústico \\
\hline & & 122: Qualidade do ar interior \\
\hline & \multirow[b]{2}{*}{ C7: Capacidade de controlo por parte dos utilizadores } & 123: Ventilação e temperatura \\
\hline & & 124: Vistas e privacidade \\
\hline & C8: Enquadramento paisagístico & 125: Ligação visual com a paisagem envolvente \\
\hline & \multirow[b]{2}{*}{ C9: Desenho passivo } & I26: Implantação e orientação \\
\hline & & 127: Sistemas passivos \\
\hline & C10: Plano de mobilidade & 128: Acessibilidades \\
\hline & & 129: Existência e acessibilidade a áreas sociais \\
\hline & & I30: Otimização espacial \\
\hline & & 131: Flexibilidade do espaço \\
\hline & C11: Flexibilidade e adaptabilidade espaciais & 132: Adaptabilidade do espaço \\
\hline \multirow{3}{*}{ A3: ECONÓMICA } & \multirow{2}{*}{ C12: Custos de ciclo de vida } & 133: Custo de investimento inicial \\
\hline & & I34: Custos de utilização \\
\hline & C13: Promoção da economia local & I35: Contratação de bens e serviços locais \\
\hline \multirow{12}{*}{ A4: TÉCNICA } & \multirow[b]{4}{*}{ C14: Sistemas de gestão ambiental } & I36: Comissionamento \\
\hline & & 137: Plano de gestão ambiental \\
\hline & & I38: Qualidade do ar interior \\
\hline & & I39: Redução da poluição sonora \\
\hline & C15: Sistemas & $\begin{array}{l}\text { 140: Eficiência dos sistemas de iluminação e } \\
\text { climatização }\end{array}$ \\
\hline & C16: Segurança & 141: Segurança dos ocupantes \\
\hline & \multirow[b]{2}{*}{ C17: Durabilidade } & 142: Materiais de elevada resistência e durabilidade \\
\hline & & 143: Seleção adequada de mobiliário \\
\hline & \multirow{3}{*}{$\begin{array}{l}\text { C18: Sensibilização e educação para a } \\
\text { sustentabilidade }\end{array}$} & 144: Formação dos ocupantes \\
\hline & & 145: Formação dos prestadores de serviços \\
\hline & & 146: Inquéritos de satisfação \\
\hline & C19: Competências na área da sustentabilidade & 147: Integração na equipa de um Avaliador Qualificado \\
\hline \multirow{5}{*}{ A5: Lugar } & C20: Comunidade local & 148: Desenvolvimento da comunidade local \\
\hline & C21: Valor cultural & 149: Enquadramento patrimonial \\
\hline & \multirow[b]{3}{*}{ C22: Acessibilidades } & 150: Acessibilidade a transportes públicos \\
\hline & & 151: Mobilidade de baixo impacte \\
\hline & & 152: Amenidades locais \\
\hline
\end{tabular}




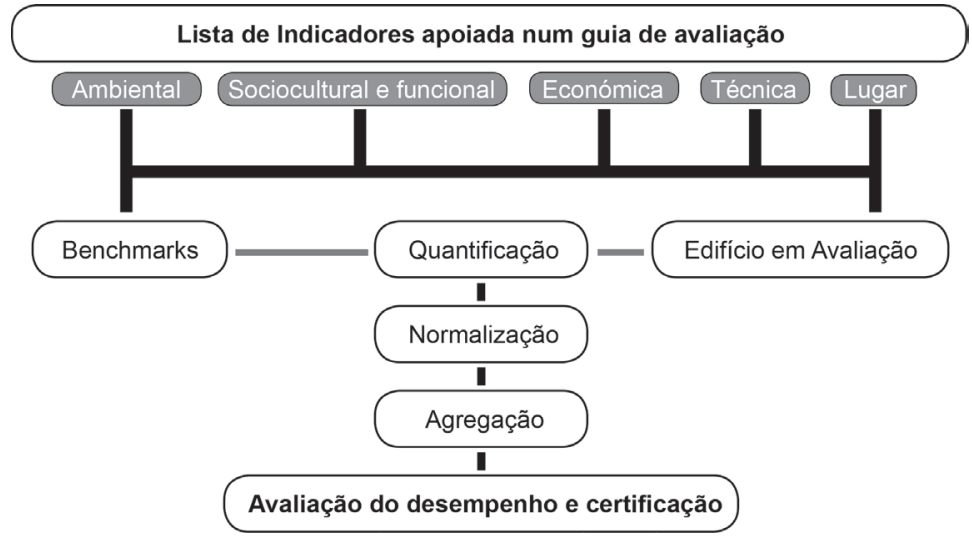

Figura 5. Esquema representativo do funcionamento do sistema de avaliação HBSATool - PT (elaborada pelo autor).

\section{UNIDADE FUNCIONAL E BENCHMARKS.}

Para alcançar os valores de referência necessários ao bom funcionamento da metodologia proposta, têm sido estudados os edifícios hospitalares nacionais, os quais foram divididos em três grupos, em função da sua Área Útil de pavimento. Optou-se por dividir os hospitais em diferentes grupos de modo a facilitar o processo de comparação dos níveis de desempenho, uma vez que hospitais com dimensões distintas apresentam normalmente um conjunto de serviços diferente e cobrem um número variável de pacientes, pelo que apresentam um impacte diferente em cada uma das cinco áreas de sustentabilidade em avaliação. Assim, a unidade funcional definida para este estudo é $1 \mathrm{~m}^{2}$ de área útil de pavimento, pelo que a quantificação dos indicadores e definição dos respetivos valores de referência (benchmarks) são realizados para esta área equivalente. Para cada um dos três grupos foram definidos os benchmarks respetivos.

Em comparação com outros parâmetros como, por exemplo, o número de camas, o ano de construção e a localização geográfica, a área útil de pavimento mostrou ser aquele que apresenta melhor correlação com o consumo de recursos de cada edifício hospitalar estudado.

Em Portugal, o Ministério da Saúde tem determinado a eficiência de cada instituição através da relação de custos utilizando a medida "doente-padrão" (ACSS, 2016). O cálculo do "doente padrão" baseia-se na transformação da atividade hospitalar, por natureza heterogénea, numa unidade de produção única de forma a possibilitar o exercício de comparação entre entidades. No entanto, importa esclarecer que o cálculo da medida "doente-padrão" não incorpora especificidades particulares e toda a carteira de serviços das entidades hospitalares, pelo que, qualquer análise de dados deve considerar uma avaliação mais profunda ao nível micro. Assim, entendendo-se que esta medida facilita apenas a comparação de custos entre edifícios, rapidamente se compreendeu que teria de se utilizar uma medida de comparação mais abrangente.

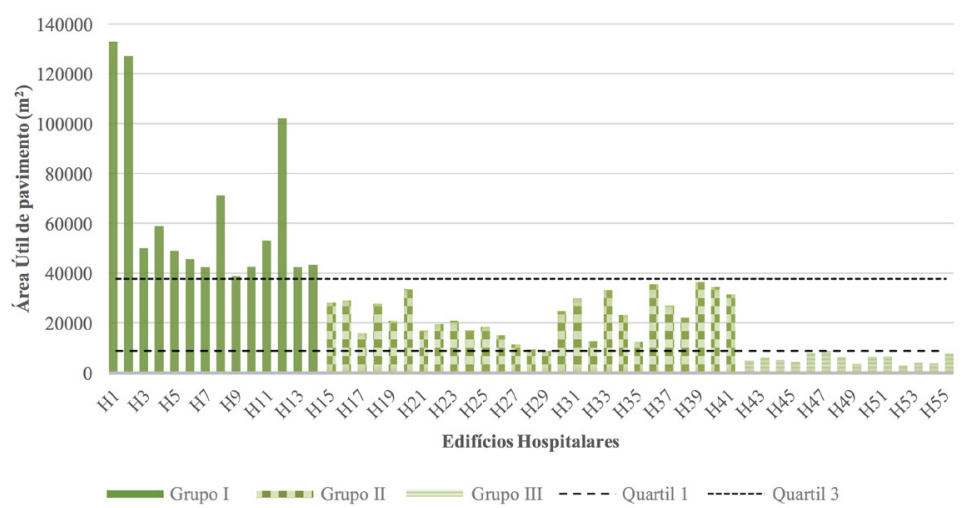

Figura 6. Divisão por grupos dos edifícios hospitalares portugueses em estudo (Castro et al., 2015: 13230).

$\mathrm{Na}$ Figura 6 apresenta-se o modo como os cinquenta e cinco edifício hospitalares portugueses foram agrupados de modo a definir os três grupos de edifícios.

Para cada grupo são estipulados valores de referência que permitirão a avaliação de edifícios hospitalares que se proponham para análise, independentemente da sua dimensão.

\section{QUANTIFICAÇÃO DOS RESULTADOS.}

A quantificação é indispensável para a comparação de soluções, agregação dos indicadores e avaliação precisa da solução. O método de quantificação a utilizar na avaliação do desempenho do edifício ao nível de cada indicador é especificado no guia de avaliação e inclui: resultados de estudos realizados anteriormente (bases de dados), normas, regulamentos e ferramentas de simulação. Por exemplo, na quantificação do consumo de energia não renovável (I2) o método a aplicar é o definido pelo Sistema Nacional de Certificação Energética dos Edifícios (Decreto-lei 118/2013).

Ao nível da quantificação das Áreas Ambiental e Técnica, a informação pode ser encontrada em diferentes fontes: Declarações Ambientais de Produto (DAPs); bases de dados gerais de Análise do Ciclo de Vida (ACV) de materiais e produtos de construção; e bases de dados genéricas de ACV de países vizinhos. Na utilização de dados de ACV, é necessário utilizar métodos de Avaliação do Impacte do Ciclo de Vida (AICV) para quantificar esses indicadores.

Em relação às Áreas sociocultural e funcional, e de lugar, os métodos de análise que devem ser utilizados para quantificar os indicadores propostos são apresentados no guia de avaliação. Finalmente, ao nível dos indicadores económicos, a metodologia é também apresentada no guia de avaliação e baseia-se na consideração de custos locais de mercado (custos relacionados com a água, gás e consumo de energia, custos de materiais, etc.). 


\section{NORMALIZAÇÃO E AGREGAÇÃO DE RESULTADOS.}

A estrutura proposta terá como base um guia de avaliação que permitirá analisar o edifício relativamente a cada Indicador proposto. No final é atribuída uma classificação geral, que pode ser apreciada ao nível de cada Indicador, Categoria ou Área. Os indicadores sujeitos à regulamentação nacional em vigor (como por exemplo o 12: Energia primária não renovável) terão de responder às exigências nacionais mínimas para poderem ser avaliados pela metodologia proposta. No entanto, tendo esta estrutura metodológica a intenção de apoiar decisões em fases preliminares de projeto, o seu guia poderá ser utilizado como manual de consulta sempre, sendo que apenas posteriormente é possível ser atribuída uma classificação final por parte do Avaliador Qualificado. À parte disto, todo e qualquer edifício de saúde Português poderá ser avaliado por esta metodologia.

Mediante a existência de indicadores qualitativos equantitativos na estrutura metodológica de avaliação proposta, torna-se necessário apresentar um método de avaliação coerente que seja transversal a todos os indicadores e equilibrado nas suas tomadas de decisão. Assim, enquanto que nos indicadores quantitativos é relativamente simples apontar valores de referência (máximos e convencionais) que permitem ao utilizador enquadrar a sua solução, nos indicadores qualitativos torna-se fundamental conhecer as expectativas de diferentes decisores de modo a definir os estremos da escala de avaliação. Deste modo, para ambas as situações, é fundamental analisar, tendo em conta cada indicador, o edificado existente e o espectável a fim de se alcançarem os valores de referência que possibilitarão a comparação. No entanto, enquanto que para o primeiro caso (como por exemplo para o 12: Energia primária não renovável) existem valores numéricos que podem ser usados, no segundo caso (como por exemplo para o I25: Ligação visual com a paisagem envolvente) é necessário recorrer a listas de créditos que permitem igualmente alcançar valores numéricos que possibilitam a comparação de casos.

Após quantificação de todos os Indicadores, é necessário proceder-se à sua normalização a fim de se possibilitar posteriormente a agregação dos mesmos para se atingir a classificação final. Deste modo é utilizada a equação 1 (Castro et al., 2015):

$$
\bar{P}_{i}=\frac{P_{i}-P_{\star i}}{P_{i}^{*}-P_{* i}} \forall_{i}
$$

$\bar{P}_{i}=$ Resultados da normalização do indicador $i$

$P_{i}=$ Resultado da quantificação de $P_{i}^{*}$

$P_{*_{i}}=$ Valores de referência do Indicador $i$, representando respetivamente a melhor prática e prática convencional

\section{CLASSIFICAÇÃO E COMUNICAÇÃO DE RESULTADOS.}

O desempenho global do edifício, o qual serve de comparação entre casos, através de uma nota final, resulta da média ponderada da soma do desempenho obtido em cada Área. Isto porque os Indicadores avaliados não apresentam a mesma importância/peso na quantificação geral de desempenho. Assim, os resultados obtidos dependem sempre do sistema de pesos definido na metodologia, o qual por sua vez reflete a existência de diferentes fatores agregados ao contexto de cada país. Por isso, não existindo ainda consenso sobre o melhor método a aplicar, é possível identificar diferenças quando se comparam pesos atribuídos por sistemas de avaliação distintos a critérios semelhantes.

Neste caso em concerto, a comunicação de resultados é feita através de uma escala de letras compreendida entre " $E$ " (o menos sustentável/abaixo do valor convencional de referência) e "A+" (o mais sustentável/acima do valor da melhor prática de referência). $O$ nível " $D$ " é o correspondente à prática convencional e o " $\mathrm{A}$ " à melhor prática (Tabela 2) (Castro et al., 2015).

\begin{tabular}{|l|l|}
\hline Escala qualitativa de sustentabilidade & Valores normalizados \\
\hline A + & $\bar{P}_{i}>1,00$ \\
\hline A (Melhor prática) & $0,70<\bar{P}_{i} \leq 1,00$ \\
\hline B & $0,40<\bar{P}_{i} \leq 0,70$ \\
\hline C & $0,10<\bar{P}_{i} \leq 0,40$ \\
\hline D (Prática convencional) & $0,00<\bar{P}_{i} \leq 0,10$ \\
\hline E & $\bar{P}_{i} \leq 0,00$ \\
\hline &
\end{tabular}

Tabela 2. Classificação final (elaborada pelo autor).

\section{CONCLUSÕES.}

Inicialmente, as ferramentas desenvolvidas para reconhecer o compromisso de um projeto com os objetivos do desenvolvimento sustentável eram apelidadas de "Metodologias de avaliação ambiental dos edifícios". No entanto, tendo por base a constante evolução e adaptação do conceito de sustentabilidade, torna-se cada vez mais apropriado a utilização do termo "Metodologias de avaliação da sustentabilidade dos edifícios". Atualmente não é possível falar-se apenas de ambiente sem se pensar nos outros pilares do conceito de Desenvolvimento Sustentável: o social e o económico. É fundamental que estas duas dimensões não se apresentem apenas como um dado adquirido, mas que assumam presença na lista de critérios de avaliação das metodologias. Neste sentido, é necessário alterar a forma de pensar os projetos de edifícios de saúde, uma vez que as questões ligadas à sustentabilidade, no seu sentido lato, não podem mais ser consideradas como um conjunto de princípios 
externos que poderão ou não ser considerados no pensamento projetual. Esta evolução corresponde a uma alteração de paradigma, no sentido da integração destes princípios com todas as outras exigências que compõe o projeto de um edifício hospitalar. É por isso que se torna importante falar de Sustainable-Effective Design (SED) e não apenas de EvidenceBased Design (EBD) ou Eco-Effective Design (EED). Por seu lado, as metodologias de avaliação da sustentabilidade de edifícios hospitalares devem ser cada vez mais especificas e abarcarem as necessidades desta tipologia de edifícios, ao invés de serem apenas adaptações das metodologias existentes para outros tipos de novas construções, o que acontece na generalidade dos casos.

Assim, reforça-se a importância do desenvolvimento da metodologia de avaliação da sustentabilidade apresentada neste artigo. Com este propósito, defende-se que o estudo e investigação no ramo das metodologias de avaliação da sustentabilidade de edifícios hospitalares deveriam estar focados em encontrar o melhor processo de avaliação (que terá de ser um misto entre avaliação qualitativa e quantitativa) e quais os indicadores que devem ser presentes à avaliação para cada categoria proposta. No caso concreto deste artigo, apresenta-se uma proposta para a estrutura e conjunto de indicadores de uma nova metodologia para a avaliação da sustentabilidade de edifícios hospitalares situados no contexto português. O desenvolvimento de estudos semelhantes noutras áreas geográficas deverá considerar as especificidades ambientais, sociais e económicas do contexto.

Os autores agradecem o financiamento atribuído pela Fundação para a Ciência e Tecnologia e o programa POPH/ FSE com a referência SFRH/BD/77959/2011.

\section{REFERÊNCIAS BIBLIOGRÁFICAS.}

ACSS. Recomendações e especificações técnicas do Edifício Hospitalar. 2011. Lisboa: ACSS - Administração Central do Sistema de Saúde, 2012.

ACSS. Guia de boas práticas para o sector da saúde. 1.3 (2013). Lisbon: ACSS, 2013.

ACSS. Portal da Saúde [online], 2016. [Dta de consulta: 14 de maio 2016]. Disponível em: http://www.portaldasaude.pt/portal.

ASHE. Green healthcare construction guidance statement. Chicago: ASHE, 2002.

BAUM, Mara e SHEPLEY, Mardelle. Eco-Effective Design \& EvidenceBased Design. Documento apresentado em: Cleanmed. Chicago, 2009, pp. 1-63.

BAUM, Mara; SHEPLEY, Mardelle; GINSBERG, Rachel e ROSTENBERG, Bill. Eco-Effective Design and Evidence-Based Design: Perceived Synergy and Conflic. HERD, 2009a, vol. 2, no. 3, pp. 56-70.
BAUM, Mara; SHEPLEY, Mardelle; ROSTENBERG, Bill e GINSBERG, Rachel. Eco-Effective Design and Evidence-Based Design: Removing Barriers to Integration. 1. San Francisco: AIA Board Knowledge Committee, 2009b.

CASTRO, Maria de Fátima; MATEUS, Ricardo e BRAGANÇA, Luís. Proposal for a Healthcare Building Sustainability Assessment (HBSA) Method. Em: World SB14 Barcelona. vol. 5. Barcelona: GBCe, 2014, pp. 607-613.

CASTRO, Maria de Fátima; MATEUS, Ricardo e BRAGANÇA, Luís. A critical analysis of building sustainability assessment methods for healthcare buildings. Environment, Development and Sustainability, 2015a, vol. 17, no. 6, pp. 1381-1412.

CASTRO, Maria de Fátima; MATEUS, Ricardo e BRAGANÇA, Luís. Estratégia para a incorporação de impactes ambientais, sociais e económicos específicos num método de Avaliação da Sustentabilidade de Edifícios de Saúde (HBSA). Em: Latin American European Conference on Sustainable Buildings and Communities. EURO ELECS 2015, Guimarães, 2015b.

CASTRO, Maria de Fátima; MATEUS, Ricardo; BRAGANÇA, Luís e SERÔDIO, Francisco. Development of Benchmarks for Operating Costs and Resources Consumption to be Used in Healthcare Building Sustainability Assessment Methods. Sustainability, 2015, vol. 7, no. 10, pp. 13222-13248.

GUENTHER, Robin e VITTORI, Gail. Sustainable healthcare architecture. 1. Nova Jérsia: John Wiley \& Sons, Inc., 2008.

GUENTHER, Robin e VITTORI, Gail. Sustainable healthcare architecture. 2. Nova Jérsia: John Wiley \& Sons, Inc., 2013

HAMILTON, Kirk. Four Levels of Evidence-Based Practice. AIA Journal of Architecture. Nova lorque: AIA, 2006, pp. 1-2.

ISO TS. ISO/AWI 21929, Building Construction - Sustainability in Building Construction - Sustainability Indicators - Part 1 - Framework for the development of indicators for buildings and core indicators. 2010. Geneva: ISO, 2010.

LEE, Wai-ling; CHAU, Chi-kwan; YIK, Francis; BURNETT, John e TSE Man Sze. On the study of the credit-weighting scale in a building environmental assessment scheme. Building and Environment, 2002, vol. 37, no. 12, pp. 1385-1396.

MATEUS, Ricardo. Avaliação da Sustentabilidade da Construção. Guimarães: Escola de Engenharia da Universidade do Minho, 2009.

PENTLAND, William. Heal Thy Self: U.S. Hospitals Are Huge Energy Hogs. Forbes [online] [Data de consulta: 17 de outubro de 2013]. Disponível em: http://www.forbes.com/sites/ williampentland/2012/08/20/u-s-hospitals-are-huge-energy-hogsbetter-light-bulbs-are-not-the-solution/.

ROBERTS, Greg e GUENTHER, Robin. Environmentally Responsible hospitals. Em: S. MARBERRY (ed.), Improving healthcare with better building design. 1. Chicago: Health Administration Press, 2006, pp. 81-107.

VAQUERO, Petra. Edifícios de saúde: Qual o caminho para a eficiência energética? Em: A. MALHEIRO (ed.), Tecno Hospital - revista de engenharia e gestão da saúde. vol. 58. Lisboa: EH ATEHP, 2013, pp. 18-20. 